\title{
OPEN Bioengineered 3D nanocomposite based on gold nanoparticles and gelatin nanofibers for bone regeneration: in vitro and in vivo study
}

\begin{abstract}
Hadi Samadian ${ }^{1}$, Hossein Khastar ${ }^{2}$, Arian Ehterami $^{3}$ \& Majid Salehi ${ }^{2,4,5} \bowtie$
The main aim of the present study was to fabricate 3D scaffold based on poly (L-lactic acid) (PLLA)/ Polycaprolactone (PCL) matrix polymer containing gelatin nanofibers (GNFs) and gold nanoparticles (AuNPs) as the scaffold for bone tissue engineering application. AuNPs were synthesized via the Turkevich method as the osteogenic factor. GNFs were fabricated by the electrospinning methods and implemented into the scaffold as the extracellular matrix mimicry structure. The prepared AuNPs and Gel nanofibers were composited by PLLA/PCL matrix polymer and converted to a 3D scaffold using thermal-induced phase separation. SEM imaging illustrated the scaffold's porous structure with a porosity range of $80-90 \%$ and a pore size range of 80 to $130 \mu \mathrm{m}$. The in vitro studies showed that the highest concentration of AuNPs (160 ppm) induced toxicity and 80 ppm AuNPs exhibited the highest cell proliferation. The in vivo studies showed that PCL/PLLA/Gel/80ppmAuNPs induced the highest neo-bone formation, osteocyte in lacuna woven bone formation, and angiogenesis in the defect site. In conclusion, this study showed that the prepared scaffold exhibited suitable properties for bone tissue engineering in terms of porosity, pore size, mechanical properties, biocompatibility, and osteoconduction activities.
\end{abstract}

Bone fractures are threatening condition which every individual may encounter in his/her life. Although simple fractures heal spontaneously with routine treatment, severe fractures required complicated therapies. The gold standard treatment for severe and extensive fractures is an autograft, which has several drawbacks despite its advantages. Hence, seeking proper alternatives is the subject of various studies and researchers. In this regard, 3D structured nanocomposites have gained a great deal of attention as the bone tissue engineering scaffold due to their remarkable properties and performance ${ }^{1-3}$. Well-designed and fabricated 3D structures can fill bone fractures, prevent collapsing surrounding tissues, and support bone cell proliferation and infiltration ${ }^{4}$. Various techniques have been developed for 3D scaffolds fabrication, such as wet electrospinning, gas-foaming, particulate leaching, rapid prototype-based techniques, and thermally-induced phase separation (TIPS) method. TIPS can be highly applicable among these methods due to its relative simplicity, flexibility, and high output ${ }^{5,6}$.

TIPS technique provides 3D structured scaffold with adjustable pore size and pore interconnectivity, which are critical for bone tissue regeneration. Moreover, this method allows the combination of materials with distinct structures to fabricate composite scaffolds. During the TIPS process, the homogenous polymer solution undergoes a phase separation under proper thermal situation resulting in the formation of polymer-rich and polymer-lean phases ${ }^{7,8}$. Using this method, it is possible to fabricate bioactive and functional 3D scaffolds with a combination of bioactive and structural materials. A sophisticated scaffold should mimic the native structure of the host tissue as much as possible. Nanofibrous scaffolds are desirable in this concept, which resembles the extracellular of native tissues ${ }^{9}$. Generally, traditional nanofibers fabrication methods such as electrospinning

\footnotetext{
${ }^{1}$ Nano Drug Delivery Research Center, Health Technology Institute, Kermanshah University of Medical Sciences, Kermanshah, Iran. ${ }^{2}$ Sexual Health and Fertility Research Center, Shahroud University of Medical Sciences, Shahroud, Iran. ${ }^{3}$ Institute for Regenerative Medicine, University of Zurich, Zurich, Switzerland. ${ }^{4}$ Department of Tissue Engineering, School of Medicine, Shahroud University of Medical Sciences, Shahroud, Iran. ${ }^{5}$ Tissue Engineering and Stem Cells Research Center, Shahroud University of Medical Sciences, Shahroud, Iran. ${ }^{\square}$ email: msalehi.te1392@gmail.com
} 


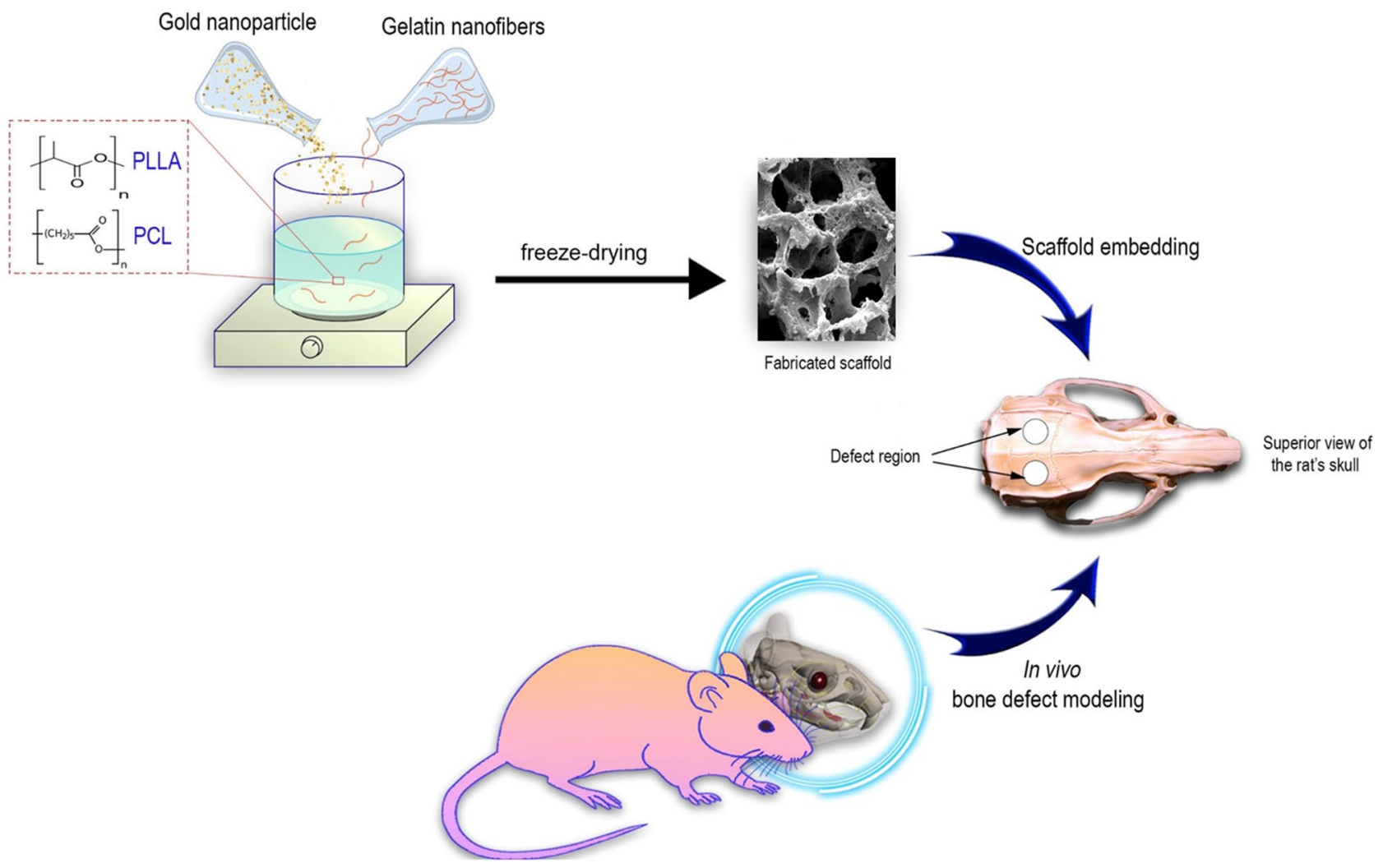

Scheme 1. Schematic representation of the study.

produce $2 \mathrm{D}$ structures that are not favorable for bone tissue engineering. An alternative approach is the combination of these methods to insert nanofibrous features into a $3 \mathrm{D}$ scaffold ${ }^{3}$.

Gelatin is a biopolymer obtains from the hydrolysis of collagen, which exhibited fascinating activity favorable for tissue engineering. Gelatin offers attractive properties such as biocompatibility, biodegradability, low cost, low immunogenicity, and acceptable solubility ${ }^{10}$. Moreover, it possesses several RGD (Arg-Gly-Asp) domains in its structure, which is beneficial for cell attachment and proliferation. Electrospun gelatin biomaterials have grabbed significant attention in regeneration medicine. Various studies utilized gelatin nanofibers in pure form or in combination with different biomaterials or structures for bone tissue engineering applications ${ }^{11,12}$. In addition to nanofibers, 3D scaffolds bearing colloidal nanoparticles (NPs) are of interest for tissue engineering applications, which offer a double advantage of having the features of both the NPs and the 3D structures ${ }^{13,14}$.

Gold NPs (AuNPs) have been widely evaluated in various biomedical fields such as cancer therapy, drug/ gene delivery, biosensors, cell tracking, and regenerative medicine ${ }^{15-17}$. AuNPs offer brilliant properties such as biocompatibility, chemical inertness and stability, high surface to volume ratio, easy synthesis, and surface modification. It is documented that AuNPs can act as the osteogenic agent and promotes bone cell differentiation and proliferation ${ }^{18-21}$. Yi et al. reported that AuNPs can elicit osteoinductive activity on mesenchymal stem cells (MSCs) via activating the p38 mitogen-activated protein kinase (MAPK) pathway ${ }^{22}$. Bone structure is a natural 3D nanocomposite comprised of collagen nanofibers and hydroxyapatite crystals ${ }^{23}$. An effective scaffold should mimic the bone structure to promote the functions of bone cells. Various types of nanocomposites have been evaluated as scaffolds for bone regeneration, such as nanofibrous nanocomposites, freeze-dried scaffolds composited with NPs or nanofibers, TIPS scaffolds composited with NPs or nanofibers, and hydrogels composited with NPs or nanofibers. To the best of our knowledge, the combination of 3D structures with both NPs and nanofibers in the form of nanocomposite has not been reported in previous studies ${ }^{24}$.

Accordingly, in the present study, we fabricated 3D scaffolds based on polylactic acid (PLLA)/Polycaprolactone (PCL) polymers containing Gel nanofibers (GNFs) and AuNPs for bone regeneration. The applied PLLA/ PCL served as the matrix, GNFs as the mimicry of the bone extracellular matrix (ECM), and AuNPs as the healing agent (Scheme 1). Different types of scaffolds have been evaluated as bone regenerating materials. 2D scaffolds, such as nanofibers, are promising due to their resemblance to the bone ECM, but they cannot fill the bone defect and properly mimic the 3D structure of the bone cells niche. On the other hand, the conventional 3D scaffolds without resemblance to native bone tissue may not properly improve bone regeneration. Accordingly, the combination of these concepts can beneficiate from the positive features of $2 \mathrm{D}$ and $3 \mathrm{D}$ scaffolds. The combination of PCL/PLLA/GNFs/AuNPs has not been evaluated in the form of a 3D scaffold for bone regeneration in animal models. The significant novelty of the present study is the combination of GNFs to mimic collagen nanofibers and AuNPs to mimic hydroxyapatite crystals to prepare a sophisticated scaffold with the most resemblance functionality to native bone structure. 


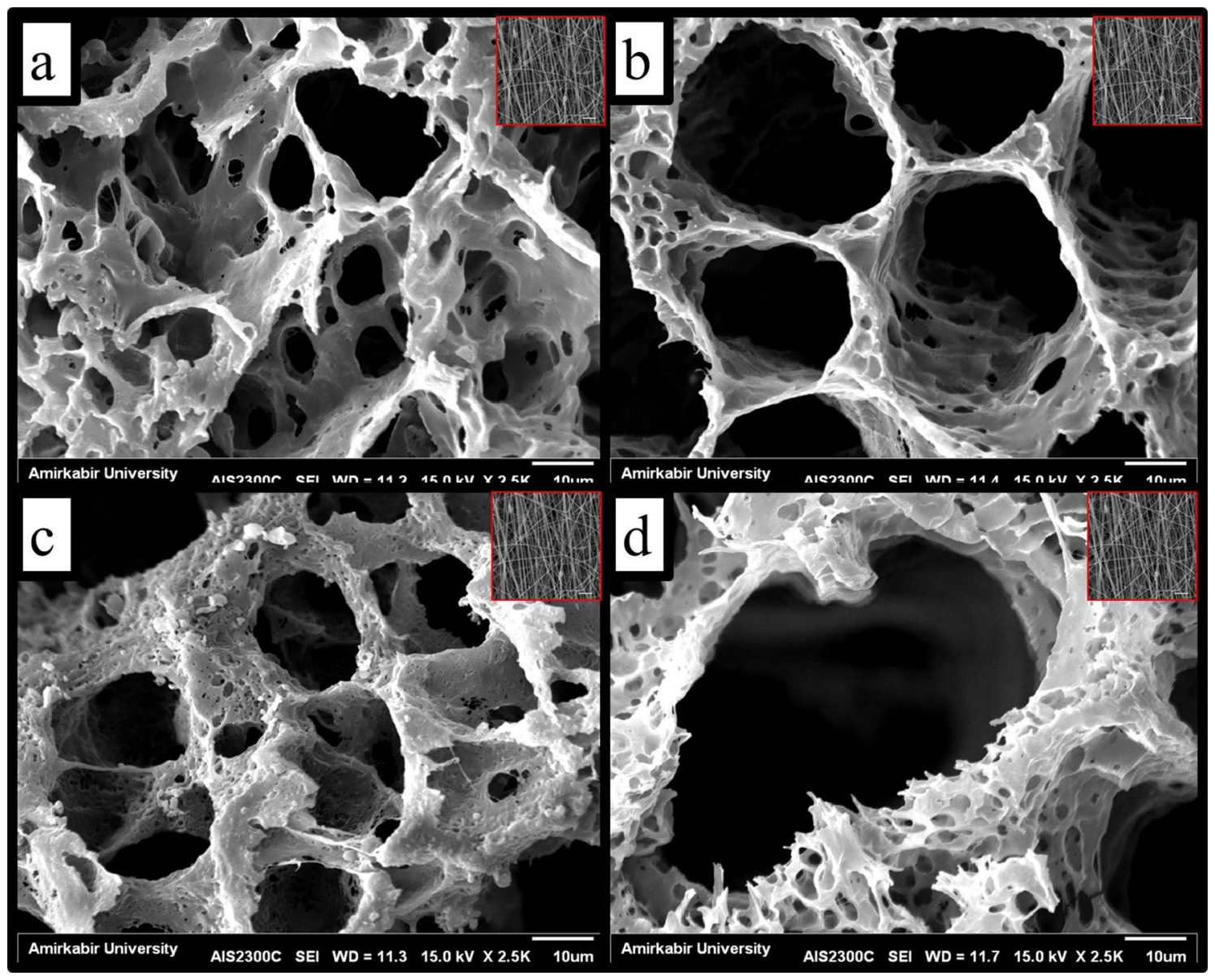

Figure 1. Scanning electron micrographs of the prepared scaffolds. (a) PCL/PLLA/GNF, (b) PCL/PLLA/GNF/ AuNPs (40 ppm), (c) PCL/PLLA/GNF/AuNPs (80 ppm), and (d) PCL/PLLA/GNF/AuNPs (160 ppm). Insert is SEM image of GNFs.

\section{Results and discussion}

Nanocomposite characterization finding. The synthesized AuNPs have an average diameter of $65.1 \mathrm{~nm}$ with a polydispersity index (PDI) of 0.526 measured with DLS. The synthesized AuNPs have zeta potential of $+9.48 \pm 0.06 \mathrm{mv}$, indicating their proper stability. The PDI of 0.526 indicate relatively proper size distribution of the synthesized NPs. SEM image of GNs was shown in Fig. 1 and the nanofibers diameter was $327 \pm 183 \mathrm{~nm}$, measured by Image J (1.47v, National Institute of Health, USA) software. Different concentrations of AuNPs $(40,80$, and $160 \mathrm{ppm})$ were added to the scaffolds and their effects on the physicochemical properties and the biological performance were evaluated. The microstructure of the prepared scaffolds was observed using SEM, and the results are presented in Fig. 1. As shown in Fig. 1, the fabricated scaffolds are highly porous (confirmed with porosity measurement assay), which is favorable for cell growth and infiltration. It is also observed that the fabricated scaffolds have interconnected pores, which is vital for the osteogenesis process. The implemented AuNPs can be both adhered onto the surface of matrix and embedded into the polymeric matrix.

The interconnected structure provides cell infiltration and migration, which results in homogenous cell growth and osteogenesis ${ }^{25}$. Moreover, the porous scaffolds with the open and interconnected pores provide vascularization in bone tissue growth, a vital requirement for proper bone tissue regeneration. The prepared scaffolds' pore size was measured using ImageJ software based on the obtained SEM images. The results showed that the pore size of PCL/PLLA/GNF, PCL/PLLA/GNF/AuNPs (40 ppm), PCL/PLLA/GNF/AuNPs (80 ppm), and PCL/PLLA/GNF/AuNPs (160 ppm) were $24.5 \pm 177,44.9 \pm 23.6,53.6 \pm 29.7$, and $75.8 \pm 33.1 \mu \mathrm{m}$, respectively. The pore size distribution was uniform in each group. Moreover, the addition of AuNPs (160 ppm) increased the porosity from $81.4 \pm 1.69$ to $88.1 \pm 2.16 \%$, which was not statistically significant $(\mathrm{p}<0.05)$. It is well established that the optimum pore size of a scaffold is in the range of $75-250 \mu \mathrm{m}$ for bone tissue regeneration ${ }^{25,26}$. Our results showed that the pore sizes of the prepared scaffolds are in the acceptable range. In addition to the pore size, the prepared scaffolds' porosity was measured using the liquid displacement method, and the results are presented in Table 1. The results showed that the scaffolds' porosity is in the range of 80 to $90 \%$, which is acceptable for bone tissue engineering applications. Previous studies showed that the incorporation of NPs increases the porosity of hydrogels. Seyyed Nasrollah et al. ${ }^{27}$ reported that the incorporation of hydroxyapatite crystals increased the porosity of polyurethane scaffold in a dose-dependent manner. They proposed that the incorporation of hydroxyapatite crystals before polymerization influenced the pore generation process. 


\begin{tabular}{|c|c|c|c|c|}
\hline & PCL/PLLA/GNF & PCL/PLLA/GNF/AuNPs (40 ppm) & PCL/PLLA/GNF/AuNPs (80 ppm) & $\begin{array}{l}\text { PCL/PLLA/GNF/AuNPs } \\
(160 \mathrm{ppm})\end{array}$ \\
\hline Porosity (\%) & $81.4 \pm 1.69$ & $83.7 \pm 2.03$ & $86.3 \pm 3.14$ & $88.1 \pm 2.16$ \\
\hline Compress modulus (MPa) & $8.65 \pm 1.18$ & $8.07 \pm 0.83$ & $7.51 \pm 1.22$ & $7.01 \pm 0.15$ \\
\hline \multicolumn{5}{|l|}{ Contact angle $\left({ }^{\circ}\right)$} \\
\hline \multicolumn{5}{|l|}{ Weight loss (\%) } \\
\hline 30 days & $30.6 \pm 1.77$ & - & - & $27.1 \pm 1.44$ \\
\hline 60 days & $41.22 \pm 2.13$ & - & - & $35.5 \pm 1.87$ \\
\hline
\end{tabular}

Table 1. The results of the fabricated scaffolds characterization.

The porosity of cancellous (or spongy) bone is in the range of 30-90\%, and a tissue engineering scaffold must be matched with its porous structure ${ }^{28,29}$. It is known that the proper vascularization and the subsequent osteogenesis take place in the macroporous scaffolds ${ }^{30,31}$. On the other hand, lower porosity promotes cell proliferation and aggregation results in enhanced osteogenesis. Microspores enhance ion exchange for apatite formation and protein adsorption due to the increased surface area, which favors cell growth and bone formation. Macrospores, provide cell infiltration, neovascularization, and proper bone ingrowth ${ }^{32-35}$.

Mechanical properties of the prepared scaffolds were assessed using the compression method according to the ASTM-D5024-95a standard, and the results are presented in Table 1. The results showed that PCL/PLA/GNF without incorporation of AuNPs has the compress modulus of $8.65 \pm 1.18 \mathrm{Mpa}$ and the addition of $160 \mathrm{ppm}$ AuNPs decreased the compress modulus to 7. $01 \pm 0.15 \mathrm{Mpa}$. The porosity and pore morphology of biomaterial strangely impact the mechanical properties. The reduced mechanical property with the incorporation of AuNPs can be attributed to the increased porosity. Although the increased porosity compromised the mechanical strength, the obtained compress modulus is in the acceptable range for bone tissue engineering ${ }^{32,36}$.

The hydrophobicity/hydrophilicity of the prepared scaffolds was measured using the water contact angle method. As shown in Table 1, the incorporation of AuNPs increased the water contact angle, indicating the scaffolds' increased hydrophobicity. Lucio et al. ${ }^{37}$ reported that the contact angle of AuNPs is $82.3^{\circ} \pm 8.0^{\circ}$ measured using freeze-fracture shadow-casting cryo-scanning electron microscopy. Accordingly, the increased water contact angle from $87.7 \pm 1.23$ to $99.2 \pm 2.03\left(^{\circ}\right)$ can be related to the partially hydrophobic nature of the synthesis AuNPs.

Weight loss measurement, as the indication of biodegradation, showed that the fabricated nanocomposites are biodegradable and $41.22 \pm 2.13 \%$ of mass weight degraded during 60 days. Moreover, it is shown that the addition of AuNPs reduced weight loss from $41.22 \pm 2.13$ to $35.5 \pm 1.87 \%$ during 60 days. This observation can be attributed to increased hydrophobicity following the incorporation of AuNPs. Biodegradation is a critical step for a proper scaffold, which makes space for the mature bone tissue formation ${ }^{38}$. The degradation rate of the scaffold must be matched with the healing rate of the injured bone tissue ${ }^{29}$. Kim et al. fabricated magnesium phosphate ceramic 3D scaffolds and observed that, at 6 weeks after implantation, the primary structure of the scaffold was broken, while the scaffold residual remained thick. In another study, Kumar et al. ${ }^{39}$ fabricated loadbearing PCL/poly (lactic-co-glycolic acid) (PLGA)-beta tri-calcium phosphate scaffolds. They observed that the increasing the PLGA concentration accelerated the degradation rate. They reported that, at 6 weeks, all PLGA present in each group was dissolved.

Cell toxicity and proliferation results. Cell toxicity and proliferation were assessed by LDH leakage assay and MTT assay kits, respectively (Figs. 2 and 3). LDH assay is colorimetric method determining the cellular cytotoxicity by measuring LDH enzyme leaked from cell cytosol through the cell membrane.

As shown in Fig. 2, the highest LDH leakage from cells was observed in PLA/PCL/GNF-160 ppm group, whereas the other groups exhibited significantly lower LDH leakage $(0.05<\mathrm{p})$. Moreover, PLA/PCL/GNF, PLA/ PCL/GNF/ AuNPs (40 ppm), and PLA/PCL/GNF/AuNPs (80 ppm) groups exhibited acceptable LDH leakage. These data imply that PLA/PCL/GNF/AuNPs $(160 \mathrm{ppm})$ scaffolds induced cytotoxic effect on cells through the damage to the cell membrane.

The proliferation of MG-63 cells on the fabricated scaffolds was measured using MTT assay (Fig. 3), along with the induced cytotoxicity. The results showed the highest cell growth was obtained by PLA/PCL/GNF/ AuNPs $(80 \mathrm{ppm})$ at $72 \mathrm{~h}$, which was statistically significant compared with the other group. Moreover, it was observed that test groups' cell proliferation was higher than the control group, except in PLA/PCL/GNF/AuNPs $(160 \mathrm{ppm})$ at $72 \mathrm{~h}$, which was lower than control. AuNPs are considered as nontoxic and biocompatible structures at the optimum concentrations. Some reports showed the concentration-dependent toxicity of AuNPs. Vecchio et al. ${ }^{40}$ reported concentration-dependent toxicity of AuNPs on Drosophila melanogaster. They showed that the observed toxic effects were due to the cellular and molecular damages induced by AuNPs at high concentrations. They proposed that the ROS generated through AuNPs may be responsible for the cellular and genotoxic effects. 


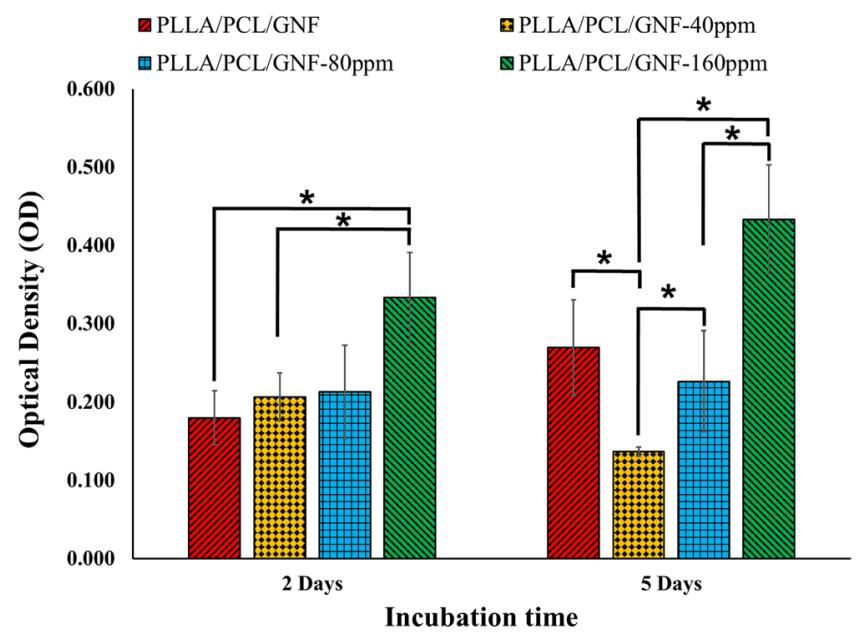

Figure 2. The cytotoxic effects of nanocomposite scaffolds on MG-63 cells measured by lactate dehydrogenase (LDH) assay. MG-63 seeded on the scaffolds with a density of 7000 cells/well in a 96-well plate and incubated for 2 and 5 days. Data represented as mean $\pm S D, n=5{ }^{*} \mathrm{p}<0.05$ (obtained by one-way ANOVA).

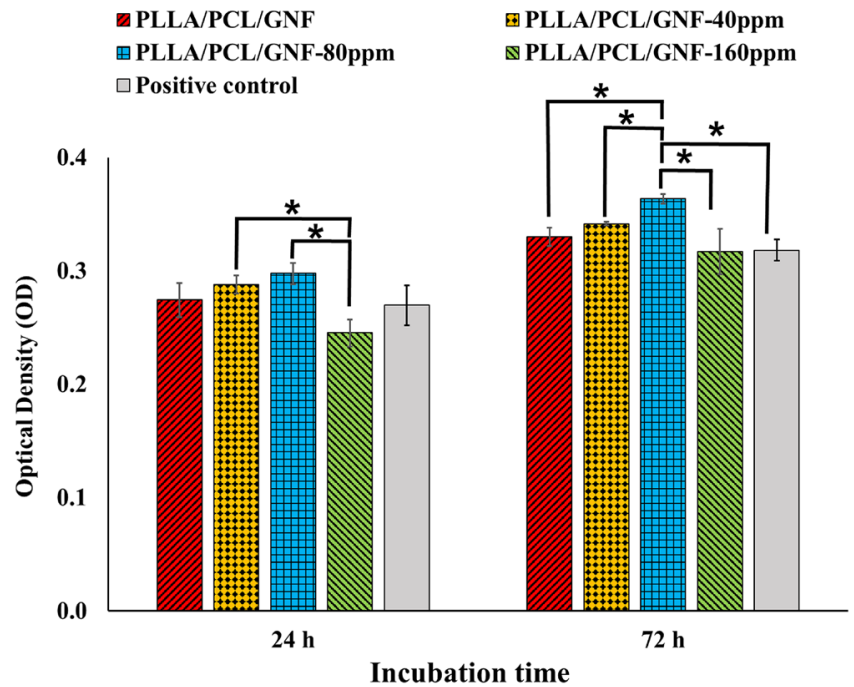

Figure 3. MG-63 cell proliferation on the fabricated scaffolds measured using the MTT assay kit at 24 and $72 \mathrm{~h}$ after cell seeding. Control: Tissue Culture Plate (TCP). Data represented as mean $\pm S D, n=3 .{ }^{*} p<0.05$ (obtained by one-way ANOVA).

Carnovale et al. ${ }^{41}$ also reported that AuNPs are biocompatible at the optimum concentration $(<100 \mu \mathrm{M})$ and the higher concentration induced toxic effects on cells.

Animal study results. The bone healing induced by the scaffolds was evaluated in the rat calvarial defect model, and the histopathological and histomorphometric results are presented in Fig. 4 and Table 2, respectively. The results showed that the defect area in the negative control (untreated defect) was filled by a loose areolar connective tissue (LACT) (Fig. 4. star) containing fibroblasts, random immature collagen fibers, and newly-formed blood vessels. The lowest bone ingrowth was also observed in this group. On the other hand, the defect treated with PLA/PCL/GNF/AuNPs (80 ppm) induced higher neo-bone formation (NB) and osteocyte in lacuna (OC) in the defect site. Moreover, the highest degree of NB and woven bone formation, as well as angiogenesis, were observed in this group. Although the scaffold remnants were also visible in the defect area (Fig. 4, arrowhead), they were relatively degraded and almost replaced with new tissues, including collagen fibers, mature bone (MB), and NB. The wettability characteristic of scaffold affects the bone regeneration with different mechanisms. Cells tend to adhere on the hydrophilic substrates and infiltrate into the hydrophilic scaffolds, subsequently regenerate bone tissue ${ }^{1}$. It is reported that the WCA above $90^{\circ}$ imply the hydrophobicity and below $90^{\circ}$ indicate the hydrophilic nature ${ }^{42}$. Our results showed that, although incorporation of AuNPs increased the WCA value, the resulted data is in the hydrophilic range, below $90^{\circ}$. The in vivo results also showed that the scaffold with the 
40X

a

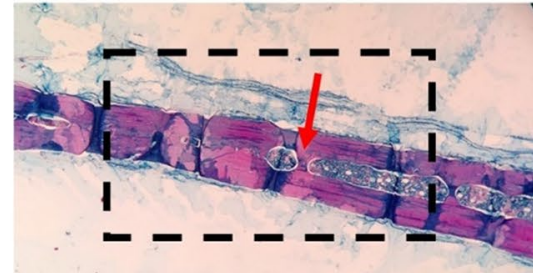

b

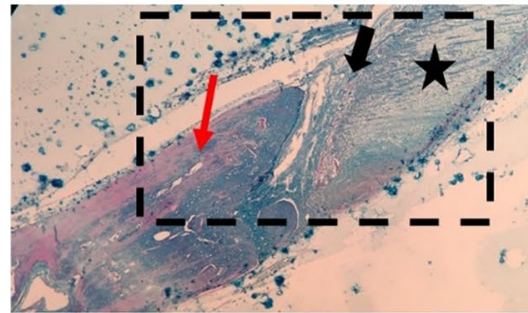

c

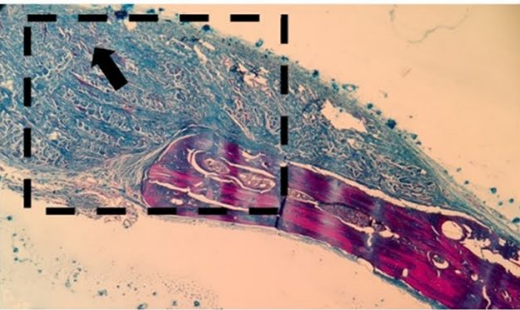

d

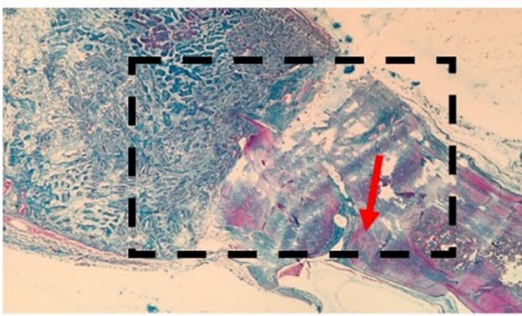

e

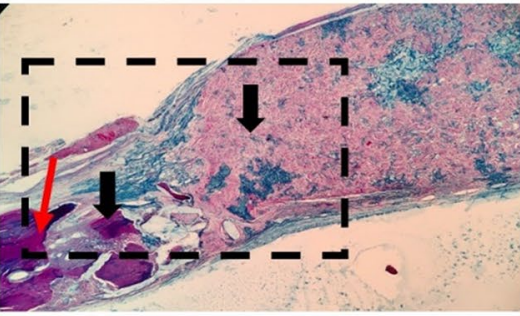

f

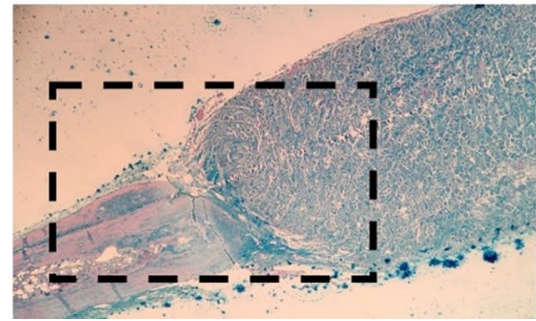

$100 X$
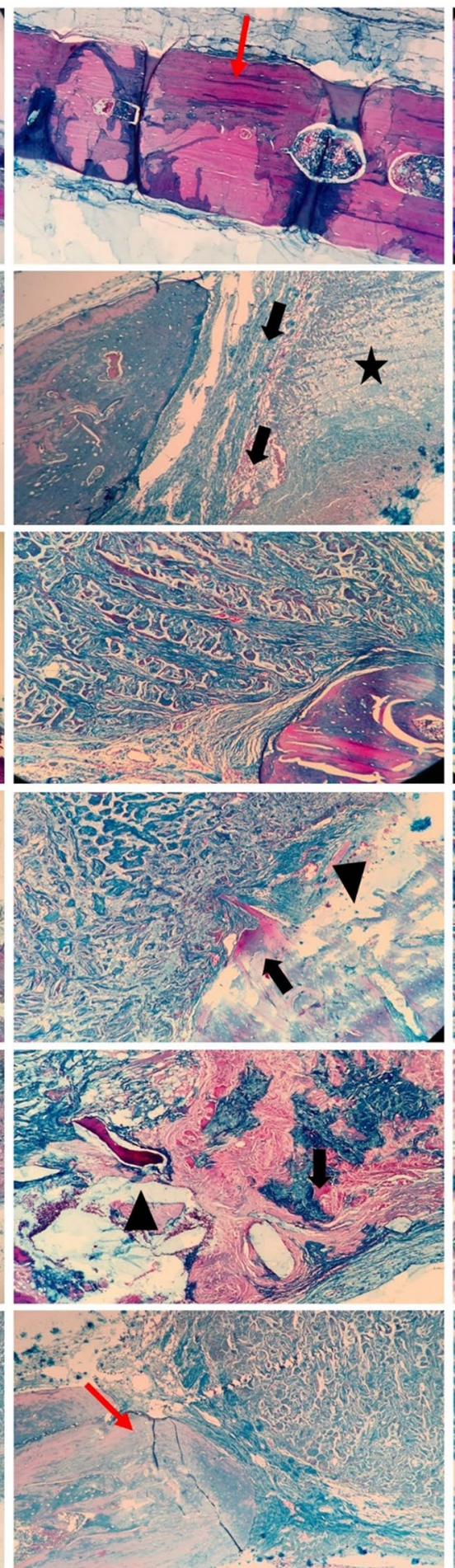

$400 X$
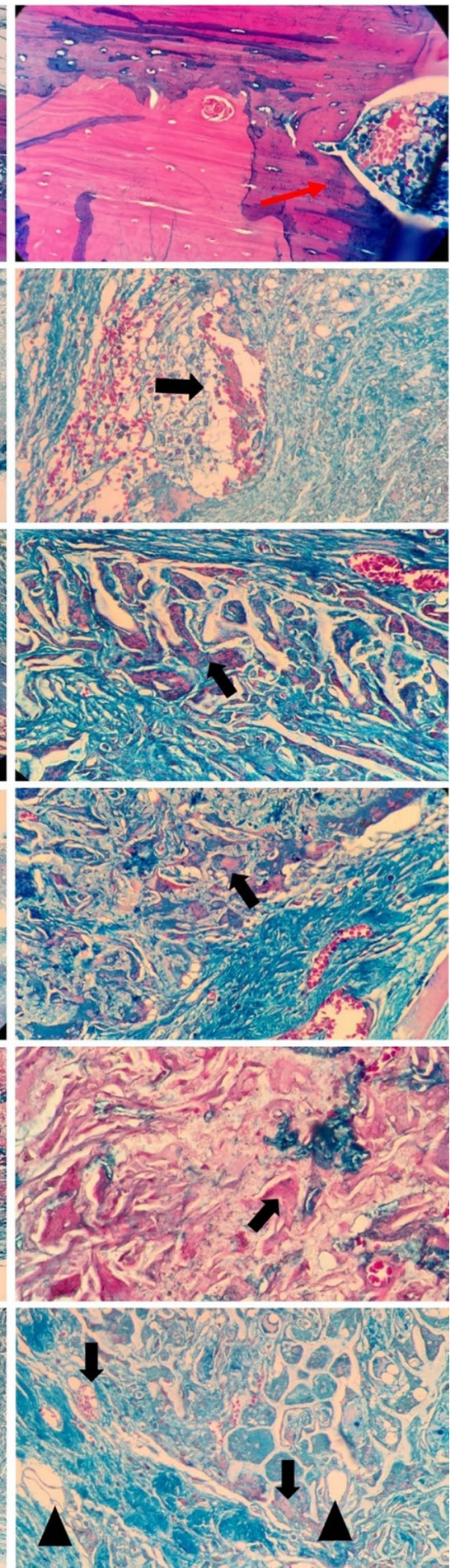

Figure 4. Histopathological sections from the calvaria bone defects and related histomorphometrical analysis (Stained with MTC). (a) Positive control, (b) Negative control, (c) PLLA/PCL/GNF, (d) PLLA/PCL/GNF/ AuNPs (40 ppm), (e) PLLA/PCL/GNF/AuNPs (80 ppm), and (f) PLLA/PCL/GNF/AuNPs (160 ppm). LACT loose areolar connective tissue (star), $N B$ new bone formation (thick arrow), $M B$ mature or old bone (red thin arrow), $S R$ scaffold remnant (arrowhead). 


\begin{tabular}{|l|c|c|l|l|l|}
\hline & Negative control & PLA/PCL/GNF & PLA/PCL/GNF/AuNPs (40 ppm) & PLA/PCL/GNF/AuNPs (80 ppm) & $\begin{array}{l}\text { PLA/PCL/GNF/AuNPs } \\
(\mathbf{1 6 0} \text { ppm) }\end{array}$ \\
\hline Fibroblast + fibrocyte & $62.10 \pm 3.55$ & $790.42 \pm 18.64$ & $93.90 \pm 16.03$ & $109.11 \pm 22.45$ & $87.09 \pm 13.10$ \\
\hline Chondroblast + chonrocyte & $102.40 \pm 5.44$ & $88.50 \pm 11.37$ & $53.73 \pm 6.74$ & $55.26 \pm 4.99$ & $67.32 \pm 7.88$ \\
\hline Osteoblast + osteocyte & $23.25 \pm 4.65$ & $51.25 \pm 18.09$ & $85.34 \pm 16.22$ & $97.60 \pm 27.16$ & $76.31 \pm 15.05$ \\
\hline Osteoclast & $3.78 \pm 1.66$ & $4.54 \pm 1.09$ & $3.31 \pm 2.14$ & $2.65 \pm 1.28$ & $4.21 \pm 0.98$ \\
\hline Osteon & $3.67 \pm 1.27$ & $5.57 \pm 1.99$ & $6.93 \pm 2.44$ & $7.55 \pm 1.69$ & $4.5 \pm 1.08$ \\
\hline
\end{tabular}

Table 2. Histomorphometric findings of bone tissue regeneration in the defect area.

highest WCA value (PLLA/PCL/GNF/AuNPs $(160 \mathrm{ppm})$, WCA of $\left.99.2 \pm 2.03^{\circ}\right)$ resulted lower bone regeneration. Accordingly, the lower bone regeneration observed in PLLA/PCL/GNF/AuNPs (160 ppm) could be attributed to the partially hydrophobic nature of the scaffold.

The histomorphometric results showed that the highest fibroblast/fibrocyte, osteoblast/osteocyte, and osteon values were obtained in defect treated with PLA/PCL/GNF/AuNPs (80 ppm). On the other hand, osteoclast cells were observed in this group, indicating more bone regeneration than bone resorption. Bone resorption osteoclast cells is the normal process of debridement after bone injuries, which reabsorb dead bone ends and make space for new bone regeneration ${ }^{43-45}$. These observations revealed that in defect treated with PLA/PCL/GNF/ AuNPs $(80 \mathrm{ppm})$, the debridement process has passed and the bone regeneration progressed. The poorest bone regeneration was observed in the negative control group, which exhibited lowest fibroblast/fibrocyte, osteoblast/ osteocyte, and osteon values. Among the test groups, the defect treated with PLA/PCL/GNF/AuNPs (80 ppm) showed the highest bone regeneration.

The osteogenic activities of AuNPs reported in several studies ${ }^{20,22,46-49}$. Liu et al. evaluated the osteogenic effects of AuNPs on murine pre-osteoblast cell line MC3T3-E1 and reported increasing of the ALP activity and expressions of Runx2, BMP-2, ALP and OCN gens. Yi et al. reported that AuNPs can elicit osteoinductive activity on mesenchymal stem cells (MSCs) via activating the p38 mitogen-activated protein kinase (MAPK) pathway ${ }^{22}$. Various studies assessed the size effect of AuNPs on the osteoinductive activity. Wan-Kyu et al. reported that $30 \mathrm{~nm}$ and $50 \mathrm{~nm}$ AuNPs were more potent osteoinductive than smaller (15 nm) or bigger $(100 \mathrm{~nm}) \mathrm{AuNP}^{50}$. Tsai et al. showed that $10 \mathrm{~nm}$ AuNPs did not exhibit osteogenic activities on MG63 osteoblast-like cells ${ }^{49}$. These results proposed that AuNPs in the size range of 30-100 nm are more beneficial for bone tissue engineering applications.

\section{Conclusion}

As an innovative and multidisciplinary approach, tissue engineering has been emerged to eliminate the limitations of conventional tissue regeneration methods. Scaffolds play a central and critical role in tissue engineering approaches and should mimic the native structure of healthy tissue as much as possible. Since bone is a natural nanocomposite, plenty of researches have been conducted to develop nanocomposite-based tissue engineering scaffolds. In the current study, we fabricated a 3D scaffold-based on PCL/PLA polymers through the TIPS method and composited the scaffold with GNFs and AuNPs. The prepared scaffolds were thoroughly characterized in terms of morphology, porosity, hydrophobicity/hydrophilicity, pore size, mechanical properties, and biocompatibility. The results showed that the fabricated nanocomposite possesses properties beneficial for bone tissue engineering. Although biodegradation is a preferred property for bone tissue engineering scaffolds and the inclusion of AuNPs within the scaffold nanocomposite seems at odds with this, the studies have shown that AuNPs do not affect the osteogenesis activities of bone cells ${ }^{49}$. Moreover, there are some reports on the biodegradation of AuNPs within cells $s^{51-53}$. It is reported that the observed degradation can be attributed to NADPH oxidase producing a high amount of ROS in the lysosome. Furthermore, we applied low amount of AuNPs $(80 \mathrm{ppm})$ in the scaffolds, which did not disturb the normal functions of cells (confirmed by hemocompatibility, cytocompatibility and animal studies). The animal study showed that PLA/PCL/GNF/AuNPs (80 ppm) scaffold induced the highest bone regeneration. For the future direction, further studies are required to clarify the fate and clearance of the applied AuNPs from within the cells or wound site. This study depicts that the combination of 3D scaffolds with zero (AuNPs) and one (GNFs) dimensional nanostructures can mimic the native structure of bone and promote the bone healing process.

\section{Materials and methods}

Materials. Gold(III) chloride trihydrate $\left(\mathrm{HAuCl}_{4} \cdot 3 \mathrm{H}_{2} \mathrm{O}, 99.9 \%\right)$, gelatin powder (bovine skin, type $\mathrm{B}$ ), poly (L-lactic) acid (PLLA, Mw $=60 \mathrm{kDa}$ ) and poly ( $\varepsilon$-caprolactone) [PCL; $\mathrm{Mw}=48-90 \mathrm{kDa}$ ] were purchased from Sigma-Aldrich (St. Louis, MO). Sodium borohydride $\left(\mathrm{NaBH}_{4}, 99 \%\right)$, cetyltrimethylammonium bromide (CTAB, 97\%), acetic acid, 1,4-Dioxane, and Dimethyl sulfoxide (DMSO) were purchased from Merck Chemicals (Darmstadt, Germany). Fetal Bovine Serum (FBS), Penicillin-Streptomycin (Pen-Strep), MTT ((3-(4, 5-dimethylthiazol-2-yl)-2.5-diphenyl-tetrazolium bromide), Dulbecco's Modified Eagle Medium: Nutrient Mixture F-12 (DMEM/F-12), and Trypsin-EDTA were purchased from Gibco (Germany). Ketamine and Xylazine were obtained from Alfasan (Woerden, Netherlands). MG-63 cell line was obtained from the National Cell Bank of Iran (NCBI), Pasteur Institute of Iran, Tehran, Iran. Male adult Wistar rats were kindly provided by Shahroud University of Medical Sciences, Shahroud, Iran. 
Synthesis of AuNPs. A chemical reduction method was used to synthesis negatively-charged AuNPs based on the previously described studies ${ }^{54,55}$. Briefly, a mixture containing an aqueous solution $(12 \mathrm{~mL}) \mathrm{of}$ $\mathrm{HAuCl}_{4} \cdot 3 \mathrm{H}_{2} \mathrm{O}(0.5 \mathrm{mM})$ and $0.5 \mathrm{ml}$ of sodium citrate $(10 \mathrm{mM})$ was stirred for $15 \mathrm{~min}$. Then, $50 \mu \mathrm{L}$ of fresh and ice-cold sodium borohydride $(0.1 \mathrm{M})$ was added to the prepared solution and mixed well for $2 \mathrm{~h}$. Finally, a $50 \mathrm{kDa}$ centrifugation filter tube was used to wash the synthesized AuNPs and the resulted nanoparticles were centrifuged at $3000 \mathrm{rpm}$ and $4{ }^{\circ} \mathrm{C}$ for $4 \mathrm{~min}$ to be concentrated.

Gelatin nanofibers (GNFs) fabrication. Gelatin nanofibers (GNFs) were fabricated based on the electrospinning technique. In this regard, gelatin powder (bovine skin, type B) was dissolved in acetic acid aqueous solution $[75 \%(\mathrm{v} / \mathrm{v})]$ to obtain a $40 \%(\mathrm{w} / \mathrm{v})$ solution. The electrospinning process was conducted using a commercial electrospinning apparatus (Fanavaran Nano-Meghyas, Tehran, Iran). The fabricated solution was loaded into a $10-\mathrm{mL}$ disposable syringe. The syringe was mounted onto the sample holder. The nozzle, a blunted 20 -gauge stainless needle, was connected to the high voltage power supply. The operating parameter, including applied voltage, the flow rate, and nozzle to collector distance, were set as $20 \mathrm{kV}, 0.40 \mathrm{~mL} / \mathrm{h}$, and $15 \mathrm{~cm}$, respectively. The produced nanofibers were collected from the aluminum wrapped collector and cross-linked using vapor of $20 \%(\mathrm{v} / \mathrm{v})$ glutaraldehyde at $37^{\circ} \mathrm{C}$ for $6 \mathrm{~h}$. The prepared nanofibers were stored in a nitrogen tank overnight, and then crushed into small pieces (GNFs).

Preparation of PCL/PLLA/GNFs/AuNPs scaffolds. A proper amount of PLLA and PCL powders were dissolved in 1,4-Dioxane and stirred for $12 \mathrm{~h}$ to obtain the solution concentration $5 \%(\mathrm{w} / \mathrm{v})$ of each polymer. The produced polymers solution was mixed with the PCL/PLLA mass ratios of 50/50 and stirred for another $6 \mathrm{~h}$. In the next step, the fabricated GNFs was added to the PCL/PLLA solution and dispersed using vigorous stirring to obtain $10 \%$ (w/w) GNFs to PCL/PLLA. Finally, various concentrations of the synthesized AuNPs (40 ppm, $80 \mathrm{ppm}$, and $160 \mathrm{ppm}$ ) were added to the PCL/PLLA/GNFs and further stirred for $24 \mathrm{~h}$. The resulted solutions were transferred and stored at $-80^{\circ} \mathrm{C}$ overnight and subsequently freeze-dried at $-54{ }^{\circ} \mathrm{C}$ for $48 \mathrm{~h}$ using a freezedrier (Telstar, Terrassa, Spain).

Characterization of the scaffolds. Scanning electron microscopy (SEM) analysis. The microstructure of the prepared PCL/PLLA/GNF and PCL/PLLA/GNF/AuNPs scaffolds were observed by means of a scanning electron microscope (SEM; KYKY Technology Development, Beijing, China) at an accelerating voltage of $20 \mathrm{kV}$ after coating with gold for 250 s using a sputter coater (SCD 004, Balzers, Germany).

Contact angle measurement. The hydrophilicity/hydrophobicity nature of the fabricated scaffolds was measure based on the sessile drop technique using a contact angle measuring system (G10, KRUSS, Germany).

Weight loss measurement. The prepared scaffolds' degradation rate was measured based on their weight loss in Phosphate Buffer Solution (PBS) ( $\mathrm{pH} 7.4$ ) during 60 days. The samples were finely cut into the shape of the disc (height and diameter of $20 \mathrm{~mm}$ and $10 \mathrm{~mm}$, respectively), carefully weighted, totally immersed in tubes filled with $10 \mathrm{~mL}$ of PBS, and incubated at $37^{\circ} \mathrm{C}$ for 60 days. At the specific time points, specimens were extracted, totally dried, weighed, and the weight loss calculated using Eq. (1).

$$
\text { Weight loss }(\%)=\frac{W_{0}-W_{1}}{W_{0}} \times 100
$$

where " $\mathrm{W}_{0}$ " is the initial weight of scaffolds and " $\mathrm{W}_{1}$ " is the weight of the dried samples after removing them from the media.

Mechanical properties. The prepared scaffolds' mechanical properties were measures based on the compression strength method according to the ASTM-D 5024-95a standard with a mechanical testing machine (Santam, Karaj, Iran). In this experiment, dry cylindrical samples of each scaffold (height and diameter of $20 \mathrm{~mm}$ and $10 \mathrm{~mm}$, respectively) were analyzed at a cross-head speed of $0.5 \mathrm{~mm} / \mathrm{min}$ at room temperature and compressed up to $75 \%$ original height. Data are described as an average of five test specimens with standard error.

Porosity assessment. The porosity of the prepared scaffolds was measured using the liquid displacement technique. Briefly, the initial volume of ethanol was measured $\left(\mathrm{V}_{1}\right), 20 \mathrm{mg}$ of each scaffold was immersed into the ethanol for one hour and the volume was measured $\left(V_{2}\right)$, and finally, the scaffold was removed from the ethanol and the volume was measured $\left(\mathrm{V}_{3}\right)$. Equation (2) was used to calculate the porosity:

$$
\text { Porosity }(\%)=\frac{V_{1}-V_{3}}{V_{2}-V_{3}} \times 100
$$

where $V_{1}$ is the initial volume of $96 \%$ ethanol, $V_{2}$ is its volume after soaking of the scaffold in ethanol and $V_{3}$ is the volume of the ethanol after the scaffold removal.

Toxicity evaluation. Cell culture studies were performed using MG-63 cell lines and the MTT assay kit was used to quantitatively measure the cell proliferation rate. The prepared scaffolds were cut spherically and put in the bottom of the 96-well plate under sterile conditions. MG-63 cells were cultured at the density of $7 \times 10^{3}$ cells on the scaffolds in DMEM/F12 culture media supplemented with $10 \%$ (v/v) FBS, 100 unit/ml penicillin, 
and $100 \mu \mathrm{g} / \mathrm{mL}$ of streptomycin incubated in a humidified incubator at $37^{\circ} \mathrm{C}$ with $5 \% \mathrm{CO}_{2}$. At each time point ( 1 and 3 days after cells seeding), the culture medium was removed from the 96 -well plate and $0.2 \mathrm{~mL}$ of MTT $\left(0.5 \mathrm{mg} / 1 \mathrm{~mL}\right.$ DMEM) was added to each well, and the cells were incubated at $37^{\circ} \mathrm{C}$ for $4 \mathrm{~h}$ in a dark place. Then, the supernatant was discarded and $150 \mathrm{~mL}$ DMSO was added to each well to dissolve the formed formazan crystals. After $10 \mathrm{~min}, 100 \mu \mathrm{L}$ from each well was transferred to a new 96-well plate and the optical density was read at $570 \mathrm{~nm}$ using Anthos 2020 microplate reader (Biochrom, Berlin, Germany).

In vivo studies. Bone defect creation. The animal studies were performed on thirty healthy adult male Wistar rats (3 months old, weighing 220-250 g) obtained from Pasteur Institute (Tehran, Iran). Animal experiments were carried out according to the Kermanshah University of Medical Sciences guidelines and approved by the university's ethical committee. The rats were randomly divided into five groups (6 rats per group): $1-\mathrm{PCL} /$ PLA/GNF scaffold without AuNPs (S-WNPs), 2-PCL/PLA/GNF/ 40 ppm AuNPs scaffold (S-40 NPs), 3-PCL/ PLA/GNF/80 ppm AuNPs scaffold (S-80 NPs), 4-PCL/PLA/GNF/160 ppm AuNPs scaffold (S-160 NPs), and 5-Negative control (defect without scaffold). The animals were then anesthetized by the IP injection of Xylazine (Alfasan, Woerden, Holland; 6-8 mg/kg) and Ketamine (Alfasan, Woerden, Holland; 70-100 mg/kg) mixture. Based on our previous study ${ }^{56}$, the spherical critical-sized defect was created in the calvaria (skull) of the rats and the fabricated scaffolds were implanted in the created defect. Briefly, the periosteum was separated and a bone defect with $7 \mathrm{~mm}$ diameter was formed by the trephine (Meisinger), at a speed rate of $1000 \mathrm{rpm}$ (the defect site was irrigated with $0.9 \%$ physiological saline to prevent bone necrosis by heat). Subsequently, the scaffolds were embedded into the defect area and the periosteum was repositioned and closed with No. 6.0 nylon suture (SUPA medical devices, Tehran, Iran). The skin was closed with No. 3.0 nylon suture (SUPA medical devices, Tehran, Iran).

Histological analysis. At the specific time point, the animals were sacrificed under anesthesia, and the harvested tissues (implanted sites) were immediately fixed in the $4 \%$ buffered formalin for $48 \mathrm{~h}$. The fixed tissues were stained with Masson's trichrome (MT) staining. A light microscope (Carl Zeiss, Thornwood, USA) equipped with a digital camera (Olympus, Tokyo, Japan) was used to capture the images and an independent pathologist interpreted the results. The histomorphometric analysis was conducted by counting and analyzing fibroblasts, fibrocytes, chondroblast, chondrocyte, osteoblasts, osteocytes, osteoclast in the defect site using computer software (Image-Pro PlusV.6). The evaluation was conducted on 400X images in six microscopic fields and the averages of different indexes $($ Mean $\pm \mathrm{SD})$ were reported.

Statistical analysis. The statistical analysis was conducted using the SPSS program, v.23 (IBM, Armonk, NY, USA) and the discriminant evaluation using a one-way ANOVA test with Tukey's multiple comparison test $(\mathrm{p}<0.05)$. All experiments were repeated thrice and samples were evaluated in triplicate. The results were expressed as the mean \pm standard errors $(S E, n \geq 3$ ), and $\mathrm{P}<0.05$ was considered as statistically significant in all evaluations.

Ethical approval. The study was carried out in compliance with the ARRIVE guidelines (https://arriveguid elines.org).

\section{Data availability}

The datasets generated during and/or analyzed during the current study are available from the corresponding author on reasonable request.

Received: 26 January 2021; Accepted: 16 June 2021

Published online: 06 July 2021

\section{References}

1. Koons, G. L., Diba, M. \& Mikos, A. G. Materials design for bone-tissue engineering. Nat. Rev. Mater. 5, 584-590 (2020).

2. Brown, J. L. \& Laurencin, C. T. Biomaterials Science 1373-1388 (Elsevier, 2020).

3. Samadian, H. et al. Electro-conductive carbon nanofibers as the promising interfacial biomaterials for bone tissue engineering. J. Mol. Liq. 298, 112021 (2020).

4. Samadian, H. et al. Sciatic nerve regeneration by using collagen type I hydrogel containing naringin. J. Mater. Sci. Mater. Med. 30, $1-12$ (2019).

5. Salehi, M. et al. Investigation of cell-free poly lactic acid/nanoclay scaffolds prepared via thermally induced phase separation technique containing hydroxyapatite nanocarriers of erythropoietin for bone tissue engineering applications. Polym. Adv. Technol. 32, 670-680 (2020).

6. Chen, P. et al. Preparation and characterization of Poly (L-lactide-co-glycolide-co- $\varepsilon$-caprolactone) scaffolds by thermally induced phase separation. J. Macromole. Sci. B 59, 427-439 (2020).

7. Zuo, J.-H. et al. Fine tuning the pore size and permeation performances of thermally induced phase separation (TIPS)-prepared PVDF membranes with saline water as quenching bath. J. Membr. Sci. 577, 79-90 (2019).

8. McKenna, E., Klein, T. J., Doran, M. R. \& Futrega, K. Integration of an ultra-strong poly (lactic-co-glycolic acid)(PLGA) knitted mesh into a thermally induced phase separation (TIPS) PLGA porous structure to yield a thin biphasic scaffold suitable for dermal tissue engineering. Biofabrication 12, 015015 (2019).

9. Massoumi, B. et al. A novel bio-inspired conductive, biocompatible, and adhesive terpolymer based on polyaniline, polydopamine, and polylactide as scaffolding biomaterial for tissue engineering application. Int. J. Biol. Macromol. 147, 1174-1184 (2020).

10. Samadian, H. et al. Electrospun cellulose acetate/gelatin nanofibrous wound dressing containing berberine for diabetic foot ulcer healing: In vitro and in vivo studies. Sci. Rep. 10, 1-12 (2020).

11. Córdoba, L. J. P. \& Sobral, P. J. Physical and antioxidant properties of films based on gelatin, gelatin-chitosan or gelatin-sodium caseinate blends loaded with nanoemulsified active compounds. J. Food Eng. 213, 47-53 (2017). 
12. Tang, Y. et al. Electrospun gelatin nanofibers encapsulated with peppermint and chamomile essential oils as potential edible packaging. J. Agric. Food Chem. 67, 2227-2234 (2019).

13. Kim, B.-S., Yang, S.-S. \& Kim, C. S. Incorporation of BMP-2 nanoparticles on the surface of a 3D-printed hydroxyapatite scaffold using an $\varepsilon$-polycaprolactone polymer emulsion coating method for bone tissue engineering. Colloids Surf. B 170, 421-429 (2018).

14. Bapat, R. A. et al. The use of nanoparticles as biomaterials in dentistry. Drug. Discov. Today 24, 85-98 (2019).

15. Zarchi, A. A. K., Amini, S. M., Salimi, A. \& Kharazi, S. Synthesis and characterisation of liposomal doxorubicin with loaded gold nanoparticles. IET Nanobiotechnol. 12, 846-849 (2018).

16. Emami, T., Madani, R., Golchinfar, F., Shoushtary, A. \& Amini, S. M. Comparison of gold nanoparticle conjugated secondary antibody with non-gold secondary antibody in an ELISA kit model. Monocl. Antibodies Immunodiagn. Immunother. 34, 366-370 (2015).

17. Esmaeili-bandboni, A. et al. Cross-linking gold nanoparticles aggregation method based on localised surface plasmon resonance for quantitative detection of miR-155. IET Nanobiotechnol. 12, 453-458 (2017).

18. Ribeiro, M. et al. Antibacterial silk fibroin/nanohydroxyapatite hydrogels with silver and gold nanoparticles for bone regeneration. Nanomed. Nanotechnol. Biol. Med. 13, 231-239 (2017).

19. Lee, D. et al. Injectable hydrogel composite containing modified gold nanoparticles: Implication in bone tissue regeneration. Int. J. Nanomed. 13, 7019 (2018).

20. Heo, D. N. et al. Enhanced bone regeneration with a gold nanoparticle-hydrogel complex. J. Mater. Chem. B 2, 1584-1593 (2014).

21. Zhang, Y. et al. Gold nanoparticles promote the bone regeneration of periodontal ligament stem cell sheets through activation of autophagy. Int. J. Nanomed. 16, 61 (2021).

22. Yi, C., Liu, D., Fong, C.-C., Zhang, J. \& Yang, M. Gold nanoparticles promote osteogenic differentiation of mesenchymal stem cells through p38 MAPK pathway. ACS Nano 4, 6439-6448 (2010).

23. Murugan, R. \& Ramakrishna, S. Development of nanocomposites for bone grafting. Compos. Sci. Technol. 65, 2385-2406 (2005).

24. Pina, S., Oliveira, J. M. \& Reis, R. L. Natural-based nanocomposites for bone tissue engineering and regenerative medicine: A review. Adv. Mater. 27, 1143-1169 (2015).

25. Tamburaci, S. \& Tihminlioglu, F. Biosilica incorporated 3D porous scaffolds for bone tissue engineering applications. Mater. Sci. Eng. C 91, 274-291 (2018).

26. Cheung, H.-Y., Lau, K.-T., Lu, T.-P. \& Hui, D. A critical review on polymer-based bio-engineered materials for scaffold development. Compos. B Eng. 38, 291-300 (2007).

27. Nasrollah, S. A. S., Najmoddin, N., Mohammadi, M., Fayyaz, A. \& Nyström, B. Three dimensional polyurethane/hydroxyapatite bioactive scaffolds: The role of hydroxyapatite on pore generation. J. Appl. Polym. Sci. 138, 50017 (2021).

28. Kim, T.-R. et al. Evaluation of structural and mechanical properties of porous artificial bone scaffolds fabricated via advanced TBA-based freeze-gel casting technique. Appl. Sci. 9, 1965 (2019).

29. Polo-Corrales, L., Latorre-Esteves, M. \& Ramirez-Vick, J. E. Scaffold design for bone regeneration. J. Nanosci. Nanotechnol. 14, 15-56 (2014).

30. Hollister, S. J. Porous scaffold design for tissue engineering. Nat. Mater. 4, 518-524 (2005).

31. Hing, K., Annaz, B., Saeed, S., Revell, P. \& Buckland, T. Microporosity enhances bioactivity of synthetic bone graft substitutes. J. Mater. Sci. Mater. Med. 16, 467-475 (2005).

32. Hutmacher, D. W., Schantz, J. T., Lam, C. X. F., Tan, K. C. \& Lim, T. C. State of the art and future directions of scaffold-based bone engineering from a biomaterials perspective. J. Tissue Eng. Regen. Med. 1, 245-260 (2007).

33. Levengood, S. K. L. \& Zhang, M. Chitosan-based scaffolds for bone tissue engineering. J. Mater. Chem. B 2, 3161-3184 (2014).

34. Wu, S., Liu, X., Yeung, K. W., Liu, C. \& Yang, X. Biomimetic porous scaffolds for bone tissue engineering. Mater. Sci. Eng. R. Rep. 80, 1-36 (2014).

35. Wang, D. et al. Highly flexible silica/chitosan hybrid scaffolds with oriented pores for tissue regeneration. J. Mater. Chem. B 3, 7560-7576 (2015).

36. Guarino, V., Causa, F. \& Ambrosio, L. Bioactive scaffolds for bone and ligament tissue. Expert Rev. Med. Devices 4, 405-418 (2007).

37. Isa, L., Lucas, F., Wepf, R. \& Reimhult, E. Measuring single-nanoparticle wetting properties by freeze-fracture shadow-casting cryo-scanning electron microscopy. Nat. Commun. 2, 1-9 (2011).

38. Velasco, M. A., Narváez-Tovar, C. A. \& Garzón-Alvarado, D. A. Design, materials, and mechanobiology of biodegradable scaffolds for bone tissue engineering. BioMed Res. Int. 2015, 1-15 (2015).

39. Kumar, A. et al. Load-bearing biodegradable polycaprolactone-poly (lactic-co-glycolic acid)-beta tri-calcium phosphate scaffolds for bone tissue regeneration. Polym. Adv. Technol. 30, 1189-1197 (2019).

40. Vecchio, G. et al. Concentration-dependent, size-independent toxicity of citrate capped AuNPs in Drosophila melanogaster. PLoS ONE 7, e29980 (2012).

41. Carnovale, C., Bryant, G., Shukla, R. \& Bansal, V. Identifying trends in gold nanoparticle toxicity and uptake: Size, shape, capping ligand, and biological corona. ACS Omega 4, 242-256 (2019).

42. Wang, W. et al. Enhancing the hydrophilicity and cell attachment of 3D printed PCL/graphene scaffolds for bone tissue engineering. Materials 9, 992 (2016).

43. Zhang, T. et al. Psoralen accelerates bone fracture healing by activating both osteoclasts and osteoblasts. FASEB J. 33, 5399-5410 (2019).

44. Gómez-Barrena, E. et al. Bone fracture healing: Cell therapy in delayed unions and nonunions. Bone 70, 93-101 (2015).

45. Hankenson, K., Gagne, K. \& Shaughnessy, M. Extracellular signaling molecules to promote fracture healing and bone regeneration. Adv. Drug Deliv. Rev. 94, 3-12 (2015).

46. Niu, C. et al. Gold nanoparticles promote osteogenic differentiation of human periodontal ligament stem cells via the p38 MAPK signaling pathway. Mol. Med. Rep. 16, 4879-4886 (2017).

47. Choi, S. Y. et al. Gold nanoparticles promote osteogenic differentiation in human adipose-derived mesenchymal stem cells through the Wnt/ $\beta$-catenin signaling pathway. Int. J. Nanomed. 10, 4383 (2015).

48. Liu, D., Zhang, J., Yi, C. \& Yang, M. The effects of gold nanoparticles on the proliferation, differentiation, and mineralization function of MC3T3-E1 cells in vitro. Chin. Sci. Bull. 55, 1013-1019 (2010).

49. Tsai, S.-W. et al. Internalized gold nanoparticles do not affect the osteogenesis and apoptosis of MG63 osteoblast-like cells: A quantitative, in vitro study. PLoS ONE 8, e76545 (2013).

50. Ko, W.-K. et al. The effect of gold nanoparticle size on osteogenic differentiation of adipose-derived stem cells. J. Colloid Interface Sci. 438, 68-76 (2015).

51. Balfourier, A. et al. Unexpected intracellular biodegradation and recrystallization of gold nanoparticles. Proc. Natl. Acad. Sci. 117, 103-113 (2020).

52. Feliu, N. et al. In vivo degeneration and the fate of inorganic nanoparticles. Chem. Soc. Rev. 45, 2440-2457 (2016).

53. Soenen, S. J., Parak, W. J., Rejman, J. \& Manshian, B. (Intra) cellular stability of inorganic nanoparticles: Effects on cytotoxicity, particle functionality, and biomedical applications. Chem. Rev. 115, 2109-2135 (2015).

54. Amini, S. M., Kharrazi, S., Rezayat, S. M. \& Gilani, K. Radiofrequency electric field hyperthermia with gold nanostructures: Role of particle shape and surface chemistry. Artif. Cells Nanomed. Biotechnol. 46, 1452-1462 (2018). 
55. Darabpour, E., Kashef, N., Amini, S. M., Kharrazi, S. \& Djavid, G. E. Fast and effective photodynamic inactivation of 4-day-old biofilm of methicillin-resistant Staphylococcus aureus using methylene blue-conjugated gold nanoparticles. J. Drug. Deliv. Sci. Technol. 37, 134-140 (2017).

56. Shahrezaee, M. et al. In vitro and in vivo investigation of PLA/PCL scaffold coated with metformin-loaded gelatin nanocarriers in regeneration of critical-sized bone defects. Nanomed. Nanotechnol. Biol. Med. 14, 2061-2073 (2018).

Acknowledgements

The authors gratefully acknowledge the research council of Kermanshah University of Medical Sciences (Grant No. 980283) for financial support.

\section{Author contributions}

H.S. and M.S. conceptualization and writing- reviewing and editing, H.K., and A.E. conducted the experiments. All authors reviewed the manuscript.

\section{Competing interests}

The authors declare no competing interests.

\section{Additional information}

Correspondence and requests for materials should be addressed to M.S.

Reprints and permissions information is available at www.nature.com/reprints.

Publisher's note Springer Nature remains neutral with regard to jurisdictional claims in published maps and institutional affiliations.

(c) (i) Open Access This article is licensed under a Creative Commons Attribution 4.0 International

License, which permits use, sharing, adaptation, distribution and reproduction in any medium or format, as long as you give appropriate credit to the original author(s) and the source, provide a link to the Creative Commons licence, and indicate if changes were made. The images or other third party material in this article are included in the article's Creative Commons licence, unless indicated otherwise in a credit line to the material. If material is not included in the article's Creative Commons licence and your intended use is not permitted by statutory regulation or exceeds the permitted use, you will need to obtain permission directly from the copyright holder. To view a copy of this licence, visit http://creativecommons.org/licenses/by/4.0/.

(C) The Author(s) 2021 